\title{
Double-blind, placebo controlled, crossover study of lamotrigine in treatment resistant partial seizures
}

\author{
G J Schapel, R G Beran, F J E Vajda, S F Berkovic, M L Mashford, F M Dunagan, \\ W C Yuen, G Davies
}

\begin{abstract}
The results of a multicentre, randomised, double-blind, placebo controlled, crossover trial of lamotrigine as add-on therapy in patients with partial seizures poorly controlled by established antiepileptic drugs (AEDs) are presented. The study consisted of two 12 week treatment periods each followed by a four week washout period. During the lamotrigine treatment phase, patients received $150 \mathrm{mg}$ or $300 \mathrm{mg}$ daily dose depending on their concomitant AEDs to achieve concentrations in the range 1-3 $\mathrm{mg} / \mathrm{L}$. Forty one patients were entered at four centres and all patients entered completed the study. There was a highly significant $(p<0.001)$ decrease in total seizure counts on lamotrigine compared with placebo. Overall, $22 \%$ of patients experienced at least a $50 \%$ reduction in the total numbers of all seizure types on lamotrigine, compared with none on placebo. When the total numbers of partial seizures (simple and complex partial) were analysed there was also a significant $(p<0.05)$ reduction in seizure counts on lamotrigine compared with placebo. When total numbers of secondarily generalised seizures were compared the trend for a reduction in this seizure type did not achieve significance $(0.05<p<0.1)$. Concomitant AED plasma concentrations were virtually unchanged. It is concluded that lamotrigine is an effective AED in the treatment of therapy-resistant partial seizures.
\end{abstract}

(F Neurol Neurosurg Psychiatry 1993;56:448-453)

Lamotrigine (3,5-Diamino-6-(2,3-dichlorophenyl)-1,2,4-triazine) is a novel antiepileptic drug (AED) chemically unrelated to the major drugs in current use. In vitro studies have suggested that lamotrigine acts at voltage-sensitive sodium channels to stabilise neuronal membranes and inhibit transmitter release, particularly glutamate. ${ }^{1}$

In healthy volunteers lamotrigine was well tolerated in doses up to $240 \mathrm{mg} /$ day with plasma levels of $1-3 \mathrm{mg} / \mathrm{L}$ which are associated with anticonvulsant effects in animal studies. ${ }^{2}$ Lamotrigine exhibited linear kinetics with no evidence of autoinduction of metabolism following seven days treatment. ${ }^{2}$ The elimination of lamotrigine is affected by concomitant AEDs. Enzyme inducing drugs such as phenytoin and carbamazepine reduce the half-life to approximately 15 hours and valproate prolongs the half-life to approximately 60 hours. Patients on both an enzyme inducer and valproate have shown an intermediate elimination half-life of approximately 24 hours similar to that seen in healthy volunteers. $^{3}$

A number of double-blind, crossover, placebo controlled studies of lamotrigine as add-on therapy have been reported but the response to lamotrigine has been variable between different studies. ${ }^{47}$ In one study the median reduction in total seizures was $59 \%$, in another it was only $18 \% .{ }^{6}$ In addition, while most have shown effects on simple or complex partial seizures, only one study showed a significant reduction in secondarily generalised seizures. ${ }^{4}$

This larger multicentre study was designed to further assess the efficacy and safety of lamotrigine in patients with partial seizures uncontrolled with established AEDs.

\section{Methods}

STUDY DESIGN

The study was a multicentre, randomised, double-blind, placebo controlled, crossover trial of lamotrigine in outpatients with refractory partial seizures, as defined by the international classification. ${ }^{8}$ One of the previous crossover studies which had included 18 patients in the analysis had failed to achieve statistical significance ${ }^{5}$ and therefore it was decided that 40-50 patients should be entered into this trial to ensure the study has sufficient power to detect a drug effect. Lamotrigine or placebo was added to an existing regime of up to two established AEDs. The trial consisted of five phases (fig 1). Patients were considered for inclusion into the trial during an initial three month baseline period. Each treatment period of 12 weeks was followed by a four week taper/washout period. Medication was tapered over the first week of the washout period and then patients were given placebo. 


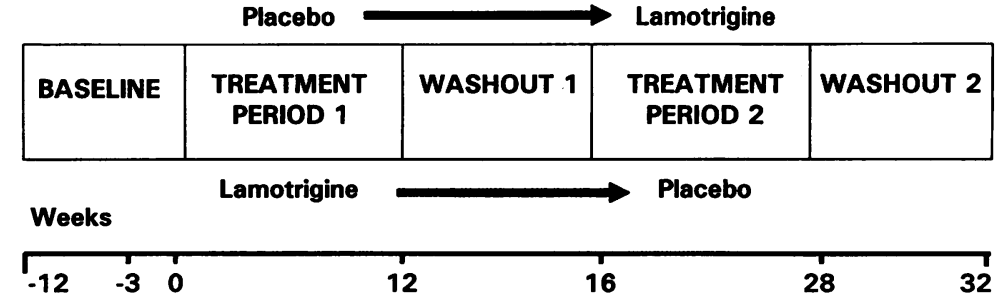

Figure 1 Study Design. two AEDs were excluded as were patients receiving valproate alone as these patients would not fit either of the dose groups.

The protocol was approved by the Institutional Ethics Committee at each Centre and all patients gave informed consent orally and in writing.

\section{PROCEDURES}

All patients had a full general physical and neurological examination together with biochemical and haematological screens and urinalysis before entry into the study. Patients were excluded if there was any abnormality on laboratory screen considered to be of clinical significance and not due to enzyme induction. Patients with any other severe organic or psychiatric disease or progressive neurological disease were also excluded.

Patients were seen at the clinic at screen and at various times throughout each treatment and taper/washout periods. At each of these visits, vital signs, seizures and any adverse experiences were recorded. Biochemistry, haematology and urinalysis together with determination of plasma AED concentrations were also carried out at most visits. In addition physical and neurological examinations were carried out at the end of each treatment period and taper/washout periods.

\section{STATISTICAL ANALYSIS}

Efficacy data

For statistical analysis, the seizure counts were accumulated to give totals for each treatment phase. The totals were calculated for all seizure types, for partial (simple and complex) seizures and for secondarily generalised (tonic-clonic) seizures. The total count of all seizure types was used as the primary efficacy parameter. The total number of days during which patients experienced at least one seizure was used as a secondary measure of efficacy.

As seizure counts were not normally distributed, log transformation was performed before data analysis (log transformations were of the form $\log (x+1))$. A parametric analysis of variance (ANOVA) was used to test for any treatment effect, centre effect, period effect or treatment $\times$ period interaction. The initial model included centre and its interactions as terms in the model but the interactions of centre with the other factors were not statistically significant and were dropped to give reduced models which were used to compute an estimate of the drug effect and the $95 \%$ confidence intervals.

\section{Safety data}

The safety of lamotrigine was evaluated by comparing haematology, biochemistry and urinalysis parameters together with adverse experience reports for the lamotrigine and placebo treatment periods. Comparisons for laboratory parameters were made using the $95 \%$ confidence intervals as well as on the basis of the number of patients with abnormal laboratory measurements. Investigators used duration of the study. Patients on more than 
a general probe to elicit adverse experiences noted by the patient. Raw terms recorded by the investigator were converted to preferred terms using a modified COSTART thesaurus for the purposes of analysis. Differences between rates of occurrence of adverse experience on placebo and lamotrigine were compared using the $95 \%$ confidence interval.

\section{Concomitant AED and lamotrigine plasma concentrations}

The mean plasma concentrations of concomitant AEDs were determined for each patient during each treatment period (where the AED was taken by at least six patients). Most of the samples were taken pre-dose and at the same time of day for individual patients. The differences between the mean concentrations for the two treatments were calculated and the confidence interval for the difference reported.

Table 1 Summary of seizure history

\begin{tabular}{|c|c|c|c|}
\hline & \multicolumn{2}{|l|}{ Treatment Sequence } & \multirow[b]{2}{*}{$\begin{array}{l}A L L \\
n=41\end{array}$} \\
\hline & $\begin{array}{l}\text { Lamotrigine/Placebo } \\
\text { Group } \\
n=20\end{array}$ & $\begin{array}{l}\text { Placebo/Lamotrigine } \\
\text { Group } \\
n=21\end{array}$ & \\
\hline \multicolumn{4}{|l|}{ Aetiology of seizures: } \\
\hline Idiopathic/unknown & 8 & 11 & 19 \\
\hline Symptomatic & 12 & 10 & 22 \\
\hline \multicolumn{4}{|c|}{ Age at onset of epilepsy (yrs) } \\
\hline Mean (SD) & $10 \cdot 5(9 \cdot 5)$ & $10 \cdot 4(10 \cdot 0)$ & $10 \cdot 4(9 \cdot 6)$ \\
\hline Range & $0-37$ & $0.5-46$ & $0-46$ \\
\hline \multicolumn{4}{|c|}{ History of status epilepticus } \\
\hline Yes & 2 & 3 & 5 \\
\hline No & 18 & 17 & 35 \\
\hline Unknown & 0 & 1 & 1 \\
\hline \multicolumn{4}{|c|}{ Number of uncontrolled seizure types: } \\
\hline 1 & 9 & 7 & 16 \\
\hline 2 & 6 & 9 & 15 \\
\hline 3 & 4 & 5 & 9 \\
\hline 4 & i & 0 & 1 \\
\hline \multicolumn{4}{|c|}{ Total baseline seizures ( 3 months pretrial) } \\
\hline Mean (SD) & $100 \cdot 7(107 \cdot 3)$ & $68 \cdot 8(86 \cdot 2)$ & $84 \cdot 3(97 \cdot 2)$ \\
\hline Range & $14-429$ & $16-406$ & $14-429$ \\
\hline \multicolumn{4}{|c|}{ Number of AEDs being taken } \\
\hline 1 & 2 & 4 & 6 \\
\hline 2 & 18 & 16 & 34 \\
\hline 3 & 0 & 1 & 1 \\
\hline
\end{tabular}

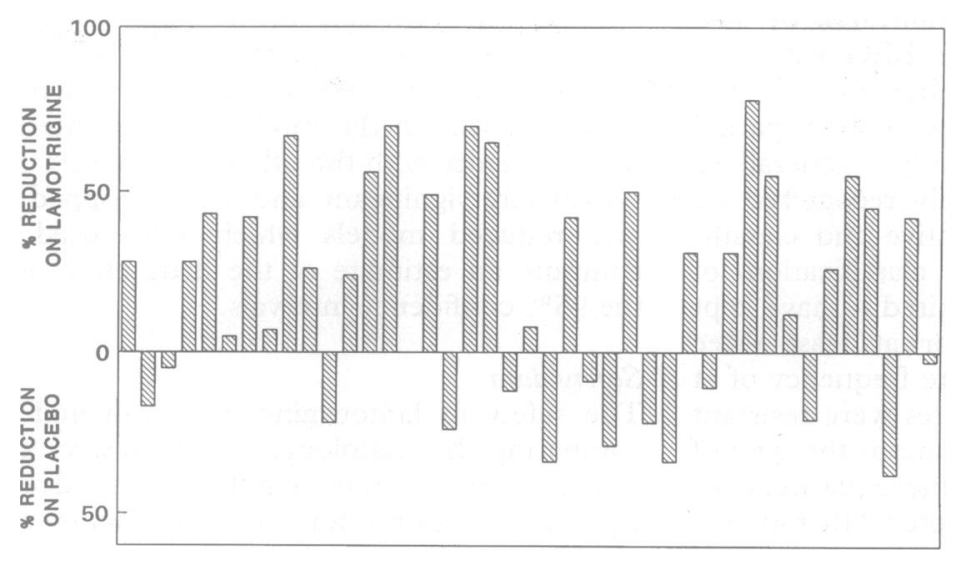

PATIENTS

Figure 2 Percentage reduction in seizure numbers on lamotrigine or placebo for individual patients. The smaller total is expressed as a percentage of the larger total for the two treatment periods.
Mean lamotrigine plasma concentrations were calculated for patients who showed a reduction in seizure count on lamotrigine and for patients who showed no change or were worse. Mean lamotrigine plasma concentrations were correlated with the mean concentration of concomitant AEDs and also with the percentage change in total seizure counts between lamotrigine and placebo treatment periods as a test of any relationship between drug concentration and clinical effect.

\section{Results}

A total of 41 patients were entered and all patients completed the study. The mean age of patients entered was 31 years with a range of 17 to 63 years. Twenty one patients were male and 20 patients were female. Aspects relating to the patients' epilepsy are summarised in table 1. From this table it can be seen that comparable types of patients were recruited to each treatment sequence group. Mean total seizure counts during the 12 week baseline period were slightly higher in the lamotrigine/placebo group but there was no significant difference in the mean total seizure counts during the study for the two groups.

\section{EFFICACY ANALYSIS}

All patients completed at least 12 weeks on lamotrigine and 12 weeks on placebo and were included in the efficacy analysis. The change in total seizure counts (the smaller total calculated as a percentage of the larger total) for individual patients were expressed as a $\%$ reduction on either lamotrigine or placebo and are presented in figure 2 for total seizures and summarised in table 2 by seizure type. For 26 out of 41 (63\%) patients there was a reduction in the total number of seizures on lamotrigine with nine $(22 \%)$ patients experiencing at least a $50 \%$ decrease in total seizure count. Fourteen patients (34\%) were better on placebo, but none experienced $50 \%$ or greater reduction in total seizure count on placebo.

When the total of simple and complex partial seizures (Type A or B) was analysed in the same way, $20 \%$ of patients experienced at least a $50 \%$ decrease in seizures on lamotrigine compared with $3 \%$ on placebo.

Seventeen patients also experienced secondarily generalised tonic-clonic seizures during the trial. For two patients this was the only seizure type recorded. When all patients were included, nine out of $19(47 \%)$ patients experienced a $50 \%$ or greater reduction in secondarily generalised seizures. Five (26\%) patients experienced a reduction in secondarily generalised seizures on placebo and three $(16 \%)$ patients had at least a $50 \%$ decrease in this seizure type. Including only patients with at least four secondarily generalised seizures per month during either the lamotrigine or placebo treatment period, the results for secondarily generalised seizures were more consistent with the effect found for simple and complex partial seizures. Five out of seven 
Table 2 Percentage change in different seizure types and number of seizure days

\begin{tabular}{|c|c|c|c|c|c|c|c|c|}
\hline & \multicolumn{2}{|c|}{$\begin{array}{l}\text { Simple and complex } \\
\text { partial seizures }\end{array}$} & \multicolumn{2}{|c|}{$\begin{array}{l}\text { Secondarily generalised } \\
\text { seizures }\end{array}$} & \multicolumn{2}{|c|}{ All seizure types } & \multicolumn{2}{|l|}{ Seizure days } \\
\hline & Lamotrigine & Placebo & Lamotrigine & Placebo & Lamotrigine & Placebo & Lamotrigine & Placebo \\
\hline $\begin{array}{l}\text { Decrease } 1-25 \% \\
\text { Decrease } 26-49 \% \\
\text { Decrease } 50-100 \% \\
\text { Total number with decrease } \\
\text { Total number with no change }\end{array}$ & $\begin{array}{r}3 \\
12 \\
8 \\
23\end{array}$ & $\begin{array}{r}8 \\
6 \\
1 \\
15\end{array}$ & $\begin{array}{r}0 \\
1 \\
9 \\
10\end{array}$ & $\begin{array}{l}1 \\
1 \\
3 \\
5\end{array}$ & $\begin{array}{r}5 \\
12 \\
9 \\
26\end{array}$ & $\begin{array}{r}10 \\
4 \\
0 \\
14\end{array}$ & $\begin{array}{r}11 \\
7 \\
9 \\
27\end{array}$ & $\begin{array}{r}8 \\
2 \\
0 \\
10\end{array}$ \\
\hline
\end{tabular}

(71\%) patients had fewer seizures (secondarily generalised) on lamotrigine and four (57\%) of these patients experienced a $50 \%$ or greater reduction in seizures. Two patients were better on placebo but none had a $50 \%$ or greater reduction in secondarily generalised seizures on placebo.

Analysis of the total number of days on which seizures were experienced between the treatment periods indicated that for $27(66 \%)$ patients there was a reduction in the number of days with seizures while on lamotrigine and nine $(22 \%)$ patients experienced at least a $50 \%$ reduction. On placebo $10(24 \%)$ patients had fewer seizure days but the reduction was less than $50 \%$. (table 2)

\section{ANOVA}

Totals of all seizure types, partial seizures (simple and complex) and secondarily generalised seizures were compared using ANOVA. Seizure counts were logarithmically transformed for the analysis of treatment effect. Data from all centres were analysed and the ANOVA model took into account any centre effect, period effect and treatment $x$ period interaction. None of these terms were significant for the total seizure count for all seizure types or for partial seizure counts, however, there was a significant centre effect for secondarily generalised seizures $(p<0.05)$ probably due to the fact that there was an imbalance in the distribution of patients experiencing secondarily generalised seizures at the four centres and the fact that the overall number of patients with secondary generalisation was small. Analysis of total counts for all seizure types showed that the treatment effect was highly significant $(p<0.001)$ and if the total number of partial seizures (simple and complex) were analysed there was also a statistically significant treatment effect in

Table 3 Rates of occurrence of adverse experiences on placebo and lamotrigine

\begin{tabular}{lcll}
\hline $\begin{array}{l}\text { Adverse } \\
\text { experience }\end{array}$ & $\begin{array}{l}\text { Incidence on } \\
\text { placebo (\%) }\end{array}$ & $\begin{array}{l}\text { Incidence on } \\
\text { lamotrigine (\%) }\end{array}$ & $\begin{array}{l}\text { 95\% CI for } \\
\text { placebo- } \\
\text { lamotrigine }\end{array}$ \\
\hline Ataxia & 5 & 17 & $(-26,2)$ \\
Dizziness & 0 & 17 & $(-29,-5)^{\star}$ \\
Nausea & 7 & 17 & $(-24,5)$ \\
Headache & 5 & 15 & $(-23,3)$ \\
Vomiting & 7 & 15 & $(-21,6)$ \\
Rash & 10 & 15 & $(-20,10)$ \\
Asthenia & 22 & 10 & $(-4,28)$ \\
Diplopia & 0 & 10 & $(-19,0)$ \\
Pain & 2 & 10 & $(-18,3)$ \\
Vision & & & $(-19,0)$ \\
$\quad$ abnormality & 0 & 10 & $(-16,1)$ \\
Rhinitis & 0 & 7 & $(-6,21)$ \\
Somnolence & 15 & 7 & \\
\hline
\end{tabular}

favour of lamotrigine $(p<0.05)$. However, when the total numbers of secondarily generalised seizures were compared the treatment effect did not achieve statistical significance $(0.05<\mathrm{p}<0.10)$.

The total numbers of days on which patients experienced seizures were also compared using ANOVA. There was no centre effect, period effect or treatment $\times$ period interaction. There was a treatment effect with a statistically significant reduction in the number of seizure days on lamotrigine $(\mathrm{p}<0.001)$.

Median reductions in seizures and confidence intervals for different seizure types were determined from the ANOVA. For all seizure types there was a median reduction of $23.9 \%$ (CI $11.5 \%, 34.6 \%$ ). The median reduction was $20.4 \%$ (CI $5.5 \%, 33.0 \%$ ) for the total of simple and complex partial seizures and $45.8 \%$ (CI $-0.01 \%, 71 \cdot 1 \%)$ for secondarily generalised seizures. The median reduction in seizure days was $21.8 \%$ (CI $10 \cdot 7,31 \cdot 5 \%)$.

\section{Concomitant AED plasma concentrations}

The mean plasma concentrations of the main concomitant AEDs; carbamazepine (30 patients), phenytoin (16 patients) and valproate (13 patients) during placebo and lamotrigine periods were calculated together with the $95 \%$ confidence intervals for the difference between the means. There was no evidence of any change in phenytoin (16.4 $\mathrm{mg} / \mathrm{L}$ on placebo and $17.0 \mathrm{mg} / \mathrm{L}$ on lamotrigine, CI $-3.2,2 \cdot 0)$ or valproate $(61.6 \mathrm{mg} / \mathrm{L}$ on placebo and $58.7 \mathrm{mg} / \mathrm{L}$ on lamotrigine, $\mathrm{CI}$ $-3 \cdot 7,9 \cdot 4)$ concentrations. There was a small but statistically significant reduction in carbamazepine concentration during the lamotrigine treatment period compared to placebo $(7.9 \mathrm{mg} / \mathrm{L}$ vs $8.3 \mathrm{mg} / \mathrm{L}$, CI $0.1,0.7)$. This may have been due to the fact that two patients had their dose of carbamazepine reduced during the lamotrigine treatment period.

\section{Lamotrigine plasma concentrations}

The lamotrigine concentrations achieved in this study ranged from 0.66 to $4.65 \mathrm{mg} / \mathrm{L}$ using the dosing regimen based on the patients' concomitant AEDs (150 mg or 300 $\mathrm{mg}$ ). The lamotrigine concentration was slightly higher in patients on valproate despite the fact that these patients received $50 \%$ of the dose given to patients on enzyme inducing AEDs only. The mean (SD) concentration was $1.95(0.75) \mathrm{mg} / \mathrm{L} \quad(\mathrm{n}=28)$ for patients on enzyme inducing AEDs only and 
$2 \cdot 37(0.72) \mathrm{mg} / \mathrm{L}(\mathrm{n}=13)$ for patients receiving valproate plus an enzyme inducer. The mean (SD) lamotrigine plasma concentration was higher for responders $[2.2(0.66) \mathrm{mg} / \mathrm{L}]$ than for non responders $[1.8(0.86) \mathrm{mg} / \mathrm{L}]$ but did not achieve statistical significance $(p$ $=0.08$ ). There was a significant correlation between mean lamotrigine plasma concentration and treatment efficacy $(p<0.05)$.

\section{SAFETY ANALYSIS}

There were 321 adverse event reports, involving 56 different adverse experiences reported throughout the trial. All patients reported at least one adverse experience. All but one of the 321 reports were classified as "not serious". Table 3 gives adverse events that were reported by at least 3 patients during the study. The $95 \%$ confidence intervals for the differences between the rates of occurrence on placebo and lamotrigine are given. Adverse experiences occurring during the taper period of the washout were included in the totals for the preceding treatment period. The confidence intervals indicate that patients on lamotrigine more frequently reported dizziness than on placebo. There were also trends for diplopia and vision abnormality to be reported more frequently on lamotrigine than on placebo, but the confidence intervals just included zero. Two patients required a reduction in dose while receiving lamotrigine because of adverse experiences, namely dizziness, headache and vomiting. There was no evidence that the lamotrigine plasma concentration was particularly high in these patients.

Six patients developed some form of rash during lamotrigine treatment. In two cases the rash was moderate in intensity and in the other cases it was considered mild. None of the rashes were considered severe. In two cases the rash was described as maculopapular and was observed 2 weeks after the start of the lamotrigine treatment period. Both of these rashes resolved without stopping lamotrigine. In the other cases the rash was reported 4 to 12 weeks after the start of the lamotrigine treatment period.

One serious adverse experience was reported for a patient who developed a CSF leak after a skull fracture due to a seizure which required surgical repair. This occurred when the patient was receiving placebo.

The median difference and $95 \%$ confidence intervals were calculated for all biochemistry and haematology parameters and there was no indication of any significant difference between lamotrigine and placebo periods. There was also no evidence of any effect of lamotrigine treatment on blood pressure, heart rate or body weight.

\section{Discussion}

In this multicentre, randomised, doubleblind, placebo controlled, crossover study, the addition of lamotrigine was associated with a significant reduction in the number of seizures experienced by patients with focal epilepsy (localisation-related epilepsy ${ }^{6}$ ) refractory to established AEDs. In this study the median reduction in the total number of all seizure types (simple and complex, partial and secondarily generalised) of $24 \%$ was very similar to the overall $27 \%$ reduction determined from a meta-analysis (92 patients) of four previous crossover placebo controlled studies with rather variable results ${ }^{4-7}$ (Wellcome Research Laboratories, personal communication). A number of reasons may explain the different responses in studies of similar design but in general it is considered that the poorer responses have been obtained in more refractory patients as indicated by factors such as the inclusion of hospitalised patients; patients with symptomatic epilepsy; patients on more than two other AEDs; patients with longer duration of epilepsy; patients with larger number of seizure types. In one previous study there was no statistically significant effect of lamotrigine on seizures but this particular study was conducted on inpatients. Seizures were documented by the nursing staff and approximately $50 \%$ of the patients were already receiving three AEDs and the same proportion of patients had a symptomatic aetiology. ${ }^{6}$

While the study reported here confirms that the addition of lamotrigine reduces the frequency of total seizures in patients with refractory focal epilepsy, the effect on secondarily generalised tonic-clonic seizures was less well defined. One previous controlled study found a significant reduction in the number of tonic-clonic seizures. ${ }^{4}$ In this particular study a large proportion of patients were experiencing secondarily generalised seizures. The present study, while including a smaller proportion of patients with secondarily generalised seizures, has provided additional evidence that lamotrigine reduces the number of this type of seizure. When patients experiencing at least four secondarily generalised seizures per month were considered, $57 \%$ experienced a $\mathbf{5 0 \%}$ or greater reduction in this seizure type on lamotrigine compared with none on placebo.

Overall in this trial, a clinically significant reduction $(>50 \%)$ in the total number of seizures of all types was found in $22 \%$ of patients, while no patients experienced this sort of improvement on placebo. For total and partial seizures the treatment effect was consistent between centres. There was no period effect or treatment $\times$ period interaction confirming the absence of any carryover between treatment phases or progressive change in patients' seizure numbers over time.

Concomitant AED plasma concentrations were monitored throughout the trial. The most common drugs were carbamazepine $(n=30)$, phenytoin $(n=16)$ and valproate $(n=13)$. While there was no effect of lamotrigine on levels of phenytoin and valproate there was a small but significant reduction in the mean carbamazepine concentration while patients were receiving lamotrigine. This was not noted in previous studies and may have 
been due to the fact that two patients in this study had their carbamazepine dose reduced while receiving lamotrigine. The decrease in the carbamazepine dose, however, does not confound the observed lamotrigine effect. This particular study demonstrated a correlation between lamotrigine plasma concentration and clinical response when expressed as a percentage reduction in total seizures. This has not been found in general in the other studies. Only the study by Loiseau et al, which used fixed doses, found a similar relationship. ${ }^{7}$ Previous studies have used "blinded" investigators who titrated the lamotrigine dose in individual patients to achieve plasma concentrations in the predicted target range 1-3 mg/L.-6 The study reported here used standard doses depending on the patients concomitant AEDs and probably gave rise to wider inter-individual variability in lamotrigine plasma concentrations. Thus it was more likely that a concentration effect relationship could be demonstrated in this study if it exists. One confounding factor which should be considered was that patients on valproate tended to have higher plasma levels than patients on enzyme inducers only, and it is possible that factors other than the lamotrigine concentration are responsible for these patients' greater response. It is also interesting that of the previous completed trials the one showing the greatest response reported one of the lowest mean lamotrigine concentrations $(1.5 \mu \mathrm{g} / \mathrm{mL}){ }^{4}$

Lamotrigine was generally well tolerated in this study and no patients were withdrawn due to adverse experiences. The most commonly reported adverse events on lamotrigine were ataxia, rash, dizziness, nausea, headache and vomiting. In two cases the investigator reduced the trial medication dose (headache and vomiting; dizziness). The incidence of rash was a little higher on lamotrigine than on placebo but this was not significant. Two patients developed a maculopapular rash (one was mild the other moderate) approximately two weeks after starting lamotrigine. In both cases the rash resolved without stopping lamotrigine. Two patients had their carbamazepine dose reduced while they were on lamotrigine due to adverse events. There did not appear to be an increase in the carbamazepine concentration and examination of a subset of patients showed no change in the epoxide metabolite at steady state. It is not possible to rule out a transient effect of lamotrigine on the epoxide metabolite. Comparison with placebo indicated that more patients reported dizziness on lamotrigine than on placebo. There was no evidence of any effect of lamotrigine on the haematological or biochemical parameters monitored.

This study has confirmed results obtained previously in controlled, crossover trials that lamotrigine is an effective antiepileptic drug in the treatment of patients with therapyresistant partial seizures and is well tolerated when given in addition to up to two other established AEDs.

The authors acknowledge the invaluable work of $\mathrm{Sr}$ Esther Lam, Sr Kylie Gebert and Research Assistant Michelle Tilley.

1 Leach MJ, Marden CM, Miller AA. Pharmacological studies on lamotrigine, a novel potential antiepileptic drug: II Neurochemical studies on the mechanism of action. Epilepsia 1986;27(5):490-7.

2 Cohen AF, Land GS, Breimer DD, Yuen WC, Winton C, Peck AW. Lamotrigine, a new anticonvulsant: pharmaPeck AW. Lamotrigine, a new anticonvulsant: pharma-
cokinetics in normal humans. Clinical Pharmacology and cokinetics in normal humans. Clinic

3 Jawad S, Yuen WC, Peck AW, Hamilton MJ, Oxley JR, Richens A. Lamotrigine: single dose pharmacokinetics and initial one week experience in refractory seizures. Epilepsy Research 1987;1:194-201.

4 Jawad S, Richens A, Goodwin G, Yuen WC. Controlled trial of lamotrigine for refractory partial seizures. Epilepsia 1989;30(3):356-63.

5 Binnie CD, Debets RMC, Engelsman, et al. Double-blind crossover trial of lamotrigine as add-on therapy in intractable epilepsy. Epilepsy Research 1989;4:222-9.

6 Sander JWAS, Patsalos PN, Oxley JR, Hamilton A, Yuen WC. A randomised double-blind placebo-controlled add-on trial of lamotrigine in patients with severe add-on trial of lamotrigine in patients

7 Loiseau P, Yuen WC, Duche B, Menager T, Arne-Bes MC. A randomised double-blind placebo-controlled crossover add-on trial of lamotrigine in patients with treatment-resistant partial seizures. Epilepsy Research 1990;7:136-145.

8 International Classification of Epileptic Seizures, 1981 Epilepsia 1981;22:489-501.

9 Dreifuss FE. Proposal for revised classification of epilepsies and epileptic syndromes. Epilepsia 1989;30: 389-99. 